\title{
S-Adenosylmethionine May Mitigate Obstructive Sleep Apnea in an Adult with Down Syndrome: A Case Report
}

\author{
Silvano Dragonieri $\mathbb{D}^{\prime}$, Giovanna Elisiana Carpagnano', Salvatore Caccamese ${ }^{2}$ \\ 'Department of Respiratory Diseases, University of Bari, Bari, Italy; ${ }^{2}$ Department of Chemical Sciences, University of Catania, Catania, Italy
}

Correspondence: Silvano Dragonieri, Department of Respiratory Diseases, University of Bari, Piazza Giulio Cesare I I, Bari, 70 I24, Italy, Tel +39080559I267, Email silvano.dragonieri@uniba.it; Salvatore Caccamese, Email scaccamese@unict.it

\begin{abstract}
Obstructive sleep apnea (OSA) is common in individuals with Down syndrome (DS). These patients are characterized by reduced levels of S-adenosylmethionine (SAMe) due to a depression of mitochondrial methylation capacity, and its implementation may improve cognitive performance. Based on the above, it is likely a beneficial effect in the treatment with SAMe also on the quality of the sleep. We report the case of an adult male with DS who received benefit of SAMe administration during OSA treatment with CPAP. We observed a significant improvement of apnea/hypopnea index (AHI), which has never been previously reported in Down individuals. AHI dropped from 51.2 to 17.2 from 0 to 49 months.
\end{abstract}

Keywords: S-adenosylmethionine, Down syndrome, sleep apnea, obstructive, oxidative stress, polysomnography

\section{Introduction}

Obstructive sleep apnea (OSA) is a common disease in adult population and it is associated with other comorbidities. ${ }^{1}$ Its incidence continues to rise and efforts should be made in investigating characteristics of OSA in special populations. ${ }^{2}$ An excellent Primer reviews the epidemiology, the mechanism and current management of this pathology. ${ }^{3}$ Down syndrome (DS) is a genetic condition resulting from the presence of an additional chromosome 21 which leads to several pathologies, in particular intellectual disability, and it is characterized by symptoms of premature aging as well as cognitive decline. ${ }^{4}$ It has been reported that OSA occurs in up to $90 \%$ of children with DS. However, adherence of therapy in this group of subjects remains low. ${ }^{5}$

Interestingly, a retrospective review was performed in children with DS and mild OSA. Anti-inflammatory medication (intranasal corticosteroids and /or montelukast) was given to a first group (29 children) and, as comparison, a second observation group (16 children) was studied. There were no significant changes in AHI and the authors conclude that several factors may explain the ineffectiveness of such medical therapy for OSA in this population. ${ }^{6}$ A recent presentation reports that adults with DS and sleep apnea had greater cortical amyloid than adults with DS and no sleep apnea. ${ }^{7}$

OSA is linked to oxidative stress by an excessive production of reactive oxygen species (ROS) and patients with more severe OSA were those with higher level of oxidative stress in their intercostal muscles. ${ }^{8}$ Alzheimer Disease (AD) has an early-onset in DS patients and changes in several biomarkers of AD in adults with DS have been demonstrated. ${ }^{9}$ In recent years interdisciplinary engagement among researchers in $\mathrm{AD}$, trisomy 21 , genetic, psychology and caregivers is leading to a better understanding of the link between $\mathrm{AD}$ and DS. ${ }^{10}$

S-Adenosylmethionine (SAMe) is found in every living cell particularly in the liver and strongly influences the central nervous system functioning. It is known that mitochondrial levels of SAMe are deeply reduced in DS due to a depression of mitochondrial methylation capacity which exposes the organelle to a more stressful condition of oxidative stress. ${ }^{11}$ Numerous preclinical and clinical studies have shown promise in the treatment of critical neuropsychiatric 
diseases including Alzheimer's disease (AD) and major depression. ${ }^{12}$ Moreover, a paper reported recently efficacy on cognitive performance of individuals with mild $\mathrm{AD}$ who received a nutraceutical formulation where $\mathrm{SAMe}$ is a major constituent. ${ }^{13}$

Based on the above, it is likely a beneficial effect in the treatment with SAMe also on the quality of the sleep, in terms of number of apnea events.

\section{Materials and Methods}

S-Adenosylmethionine was obtained from Life Extension Europe (Venlo, The Netherlands) as tablets of $200 \mathrm{mg}$ and it is underivatized. Polysomnography was recorded with Vitalnight (VitalAire Italia, Bari, Italy) or SOMNOtouchRESP (Somnomedics, Kragerø, Norway). CPAP appliance was a Blunotte from MedicAir Italia (Pogliano Milanese, MI, Italy).

\section{Results and Discussion}

We hereby report the case of an adult male with Down syndrome (age 51 years) who received benefit of SAMe administration during OSA treatment with Continuous Positive Air Pressure (CPAP). The BMI of the subject was $22.7 \mathrm{~kg} / \mathrm{m}^{2}$, he did not report cardiac comorbidities and had no history of epilepsy and snoring. L-Thyroxine was the only medicament regularly consumed by the subject. Supplementation with one capsule per day of green tea extract, providing $326 \mathrm{mg}$ of epigallocatechin-3-gallate (a polyphenol which counteracts oxidative stress and mitochondrial energy deficit in DS individuals ${ }^{14,15}$ ) was already consumed before OSA treatment.

SAMe was administered as daily tablets of $200 \mathrm{mg}$. To reduce the risk of increased blood levels of homocysteine, daily prefolic acid $15 \mathrm{mg}$ (for the first 2 months, then folic acid $400 \mu \mathrm{g}$ ), vitamin $\mathrm{B}_{12} 500 \mu \mathrm{g}$, vitamin $\mathrm{B}_{1} 250 \mathrm{mg}$ and vitamin $\mathrm{B}_{6} 250 \mathrm{mg}$ were also associated.

Nocturnal cardio-respiratory monitorings were always performed at room air without ventilation 6-7 hours per night at home and results were subsequently scored by a registered sleep technologist.

Table 1 shows parameters of CPAP therapy during SAMe administration.

Data were obtained from annual outpatient check up. The large interval between measurements at 32 and 49 months was due to lack of the annual visit due to COVID-19 restrictions. Evaluation time in polysomnography (PSG) was an average of 7 hours.

At baseline, patient had severe OSA with AHI of 51.2. Then, he started CPAP treatment at $9.5 \mathrm{~cm} \mathrm{H}_{2} \mathrm{O}$ and, at the same time, supplementation of SAMe as indicated above. After 13 months, a new PSG revealed that OSA became moderate with AHI of 22.8. He continued treatment with CPAP for additional 28 months until one day before PSG at room air without ventilation and, remarkably, the OSA became mild with AHI 13.3. However, this good result was probably due to a memory effect. Thus, after 32 months from baseline, CPAP treatment was stopped 4 days before titration at room air without ventilation and OSA remained moderate with AHI 21.8. This improvement was confirmed by last titration at 49 months when an AHI 17.2 was observed. Remarkably, the sleep posture did not affect the AHI

Table I Parameters of CPAP Treatment During SAMe Administration

\begin{tabular}{|c|c|c|c|c|c|}
\hline & 0 & 13 Months & 28 Months & 32 Months & 49 Months \\
\hline $\mathrm{AHI} / \mathrm{h}$ & 51.2 & 22.8 & 13.3 & 21.8 & 17.2 \\
\hline Longer apnea (sec) & 44 & 44 & 38 & 35 & 69 \\
\hline Average apnea (sec) & 21 & 17 & 20 & 16 & 31 \\
\hline Time (\%) in supine pos. & & 23 & 25.3 & 12 & 47.4 \\
\hline Average $\mathrm{SpO}_{2}$ & 91 & 94 & 96 & 95 & 97 \\
\hline $\mathrm{SpO}_{2}<90$ time $\%$ & 2 & 14.9 & 0.2 & 2.6 & 0 \\
\hline CPAP $\left(\mathrm{cmH}_{2} \mathrm{O}\right)$ & - & 9.5 & $9.5^{\mathrm{a}}$ & $10^{\mathrm{b}}$ & $10^{\mathrm{b}}$ \\
\hline
\end{tabular}

Notes: ${ }^{a}$ Until I day before titration; ${ }^{b}$ until 4 days before titration. 
value. Indeed, although at 49 months sleeping in supine position was $47.4 \%$ time, much more than in previous PSG, the AHI was reduced.

Spontaneous improvement of AHI has been previously described with a much higher frequency in children than adult patients in a normal population. ${ }^{16,17}$ The association found by us between SAMe and OSA severity has never been reported in Down individuals.

It is likely that administration of SAMe may have considerably reduced the apnea/hypopnea index from a very severe starting condition. Oxidative stress associated with OSA is due to a reduced oxygen availability during the apneic events. $^{3}$ Indeed, SAMe, as a potent methyl donor, counteracts too high oxidative stress in the respiratory muscles of patients. ${ }^{8}$ The effect of this compound on cultured neuroblastoma SH-SYSY cell has been demonstrated through attenuation of the oxidative stress induced by amyloid- $\beta .^{18}$

Hence, we conclude that administration of this natural compound synthesized in the liver in the homocysteine metabolism, and used as a common dietary supplement or as a treatment option in depression, might be effective in mitigating OSA in a DS adult and this means improvement in the quality of life. Deficit in memory and attention occurs in this subject and prodromal Alzheimer's disease was inferred from neuropsychological tests Leiter 3 and RBANS.

In our opinion these data warrant further case-control studies on larger number of patients with DS to confirm these preliminary findings.

\section{Ethics and Consent Statements}

Institutional approval was not required to publish the current case report. Due to his inability in provide consent to publication of the present case report, patient's legal guardian signed it.

\section{Disclosure}

SC personal involvement (father of the patient with DS) has not influenced the results of CPAP. The authors declare no other conflicts of interest for this work.

\section{References}

1. Osman AM, Carter SG, Carberry JC, Eckert DJ. Obstructive sleep apnea: current perspectives. Nat Sci Sleep. 2018;10:21-34. doi:10.2147/NSS. S124657

2. Dragonieri S, Bikov A. Obstructive sleep apnea: a view from the back door. Medicina. 2020;56(5):208-210. doi:10.3390/medicina56050208

3. Lévy P, Kohler M, McNicholas WT, et al. Obstructive sleep apnoea syndrome. Nat Rev Dis Primers. 2015;1:15015. doi:10.1038/nrdp.2015.15

4. Antonarakis SE, Skotko BG, Rafii MS, et al. Down syndrome. Nat Rev Dis Primers. 2020;6(1):9. doi:10.1038/s41572-019-0143-7

5. Horne RS, Wijayaratne P, Nixon GM, Walter LM. Sleep and sleep disorders breathing in children with Down syndrome: effects on behaviour, neurocognition and the cardiovascular system. Sleep Med Rev. 2019;44:1-11. doi:10.1016/j.smrv.2018.11.002

6. Yu W, Sarber KM, Howard JM, et al. Children with Down syndrome and mild OSA: treatment with medication versus observation. $J$ Clin Sleep Med. 2020;16(6):899-906. doi:10.5664/jcsm.8358

7. Lao PJ, Zimmerman ME, Gutierrez J, et al. Sleep apnea may be a modifiable target in the development of cerebrovascular disease and cortical amyloid in older adults with Down syndrome. 4th Trisomy 21 Research Society International Conference, Virtual; June 8-10; 2021.

8. Barreiro E, Nowinski A, Gea J, Sliwinski P. Oxidative stress in the external intercostal muscles of patients with obstructive sleep apnoea. Thorax. 2007;62(12):1095-1101. doi:10.1136/thx.2006.069963

9. Fortea J, Vilaplana E, Carmona-Iraqui M, et al. Clinical and biomarkers changes of Alzheimer's disease in adults with Down syndrome: a crosssectional study. Lancet. 2020;395(10242):1988-1997. doi:10.1016/S0140-6736(20)30689-9

10. Snyder HM, Bain LJ, Brickman AM, et al. Further understanding the connection between Alzheimer's disease and Down syndrome. Alzheimers Dement. 2020;16(7):1065-1077. doi:10.1002/alz.12112

11. Infantino V, Castegna A, Iacobazzi F, et al. Impairment of methyl cycle affects mitochondrial methyl availability and glutathione level in Down's syndrome. Mol Genet Metab. 2011;102(3):378-382. doi:10.1016/j.ymgme.2010.11.166

12. Gao J, Cahill CM, Huang X, et al. S-adenosylmethionine and transmethylation pathways in neuropsychiatric diseases throughout life. Neurotherapeutics. 2018;15(1):156-175. doi:10.1007/s13311-017-0593-0

13. Remington R, Bechtel C, Larsen D, et al. Maintenance of cognitive performance and mood for individuals with Alzheimer's disease following consumption of a nutraceutical formulation: a one-year, open-label study. J Alzheimers Dis. 2016;51(4):991-995. doi:10.3233/JAD-151098

14. De la Torre R, De Sola S, Hernandez G, et al. Safety and efficacy of cognitive training plus epigallocatechin-3-gallate in young adults with Down's syndrome (TESDAD): a double-bind, randomized, placebo-controlled, phase 2 trial. Lancet Neurol. 2016;15(8):801-810. doi:10.1016/S14744422(16)30034-5

15. Vacca RA, Valenti D, Caccamese S, Daglia M, Braidy N, Nabavi SM. Plant polyphenols as natural drugs for the management of Down syndrome and related disorders. Neurosci Biobehav Rev. 2016;71:865-877. doi:10.1016/j.neubiorev.2016.10.023

16. Chervin RD, Ellenberg SS, Hou X, et al. Prognosis for spontaneous resolution of OSA in children. Chest. 2015;148(5):1204-1213. doi:10.1378/ chest.14-2873 
17. Slonkova J, Bar M, Nilius P, Berankova D, Salounova D, Sonka K. Spontaneous improvement in both obstructive sleep apnea and cognitive impairment after stroke. Sleep Med. 2017;32:137-142. doi:10.1016/j.sleep.2016.11.024

18. Li Q, Cui J, Fang C, Liu M, Min G, Li L. S-adenosylmethionine attenuates oxidative stress and neuroinflammation induced by amyloid- $\beta$ through modulation of glutathione metabolism. J Alzheimers Dis. 2017;58(2):549-558. doi:10.3233/JAD-170177

\section{Publish your work in this journal}

Nature and Science of Sleep is an international, peer-reviewed, open access journal covering all aspects of sleep science and sleep medicine, including the neurophysiology and functions of sleep, the genetics of sleep, sleep and society, biological rhythms, dreaming, sleep disorders and therapy, and strategies to optimize healthy sleep. The manuscript management system is completely online and includes a very quick and fair peer-review system, which is all easy to use. Visit http://www.dovepress.com/testimonials.php to read real quotes from published authors.

Submit your manuscript here: https://www.dovepress.com/nature-and-science-of-sleep-journal 